\title{
(C) OPEN ACCESS \\ Concussion management in combat sports: consensus statement from the Association of Ringside Physicians
}

\author{
John Neidecker, ${ }^{1,2}$ Nitin K Sethi, ${ }^{3}$ Randolph Taylor, ${ }_{1}^{4,5}$ Raymond Monsell, ${ }^{6,7}$ Don Muzzi, ${ }^{8,9}$ \\ Bruce Spizler, ${ }^{10}$ Larry Lovelace, $^{11}$ Edmund Ayoub, ${ }^{12}$ Rick Weinstein, ${ }^{13}$ \\ Joseph Estwanik, ${ }^{14}$ Patricio Reyes, ${ }^{15}$ Robert C Cantu, ${ }^{16,17}$ Barry Jordan, ${ }^{18}$ \\ Margaret Goodman, ${ }^{19}$ John W Stiller, ${ }^{20,21}$ Jonathan Gelber, ${ }^{22,23}$ Robert Boltuch, ${ }^{24}$ \\ Domenic Coletta, ${ }^{25}$ Angela Gagliardi, ${ }^{26}$ Stephen Gelfman, ${ }^{27}$ Patrick Golden, ${ }^{28}$ \\ Nicholas Rizzo, ${ }^{29}$ Paul Wallace, ${ }^{30}$ Allan Fields, $^{31}$ Calvin Inalsingh ${ }^{32}$
}

For numbered affiliations see end of article.

Correspondence to Dr John Neidecker, Department of Sports Medicine, Orthopaedic Specialists of North Carolina, Raleigh, NC 26614, USA; neidy13@yahoo.com

Received 8 November 2017 Revised 4 July 2018 Accepted 4 July 2018 Published Online First 26 July 2018

\section{Check for updates}

(c) Author(s) (or their employer(s)) 2019. Re-use permitted under CC BY-NC. No commercial re-use. See rights and permissions. Published by BMJ.

To cite: Neidecker J, Sethi NK, Taylor R, et al. Br I Sports Med 2019:53:328-333

\section{ABSTRACT}

Various organisations and experts have published numerous statements and recommendations regarding different aspects of sports-related concussion including definition, presentation, treatment, management and return to play guidelines. ${ }^{1-7}$

To date, there have been no written consensus statements specific for combat sports regarding management of combatants who have suffered a concussion or for return to competition after a concussion. In combat sports, head contact is an objective of the sport itself. Accordingly, management and treatment of concussion in combat sports should, and must, be more stringent than for non-combat sports counterparts.

The Association of Ringside Physicians (an international, non-profit organisation dedicated to the health and safety of the combat sports athlete) sets forth this consensus statement to establish management guidelines that ringside physicians, fighters, referees, trainers, promoters, sanctioning bodies and other healthcare professionals can use in the ringside setting. We also provide guidelines for the return of a combat sports athlete to competition after sustaining a concussion. This consensus statement does not address the management of moderate to severe forms of traumatic brain injury, such as intracranial bleeds, nor does it address the return to competition for combat sports athletes who have suffered such an injury. These more severe forms of brain injuries are beyond the scope of this statement. This consensus statement does not address neuroimaging guidelines in combat sports.

PREAMBLE: DEVELOPMENT OF THIS STATEMENT This consensus statement expresses a collaborative effort among the Association of Ringside Physicians (ARP) board, emeritus board and some ARP subject matter expert members. The statement was originally made available for public view on the ARP website (http://www.ringsidearp.org/) in April of 2017. An extensive literature search including but not restricted to MEDLINE (http://www.ncbi.nlm. nih.gov/pubmed/), Cochrane Reviews (http://www. cochrane.org/reviews/) and non-indexed peer-reviewed articles published in online medical journals was performed regarding combat sports and concussion. Unfortunately, significant studies/articles/ information in combat sports regarding concussion are lacking. Hence, the majority of this statement is an amalgamation of current recommendations on concussion in non-combat sports with the current best practices in combat sports and the collective expertise and experience of its authors having provided ringside medical coverage over many years. Initially, the later mentioned guidelines were created and broadly agreed on by the entire ARP board. A small group of the authors (JN and NS), who subspecialise in sports-related concussion, was then delegated the task to compose the first draft of the accompanying statement. Once the first draft was completed, the remaining authors (RT, RM, DM, PR, RC, BJ, MG and JS), who sub-specialise in neurology, neuroanaesthesiology and neurosurgery, first edited the document. All remaining authors (BS, LL, EA, RW, JE, JG, RB, DC, AG, SG, PG, NR, $\mathrm{PW}, \mathrm{AF}, \mathrm{CI}$ ) including those who do not specialise in neurosciences, then performed final edits. The final draft of the manuscript was reviewed and approved by all authors. Since the original final draft of April 2017, the statement has been revised to this current document. The revised document is based on consensus statements, guidelines and other related articles subsequently published. The revision has followed the same editing process as the original 2017 document.

\section{DEFINITIONS AND CLARIFICATIONS Concussion}

The fifth international consensus statement on concussion in sport defines concussion as '[a subset of mild] traumatic brain injury induced by biomechanical forces" ${ }^{5}$ Several common features that may be used in clinically defining the nature of a concussive head injury can be found in box 1 .

\section{Knock out (KO)}

The definition of a $\mathrm{KO}$ differs depending on the combat sport. In boxing/kickboxing, it is defined as the failure of a combatant to get up after being knocked down by his or her opponent before the referee counts to 10 . Furthermore, in boxing/kickboxing, the referee can waive off a count of 10 and stop the bout via $\mathrm{KO}$ if it is clear the combatant will not be able to get up (eg, loss of consciousness (LOC)). KO can happen due to head blows or body blows. In the latter, a $\mathrm{KO}$ is most likely 
Box 1 Definition of concussion from the consensus statement on concussion in sport: the 5 th International Conference on Concussion in Sport held in Berlin, October $2016^{5}$

1. Concussion may be caused either by a direct blow to the head, face, neck or elsewhere on the body with an impulsive forces transmitted to the head.

2. Concussion typically results in the rapid onset of shortlived impairment of neurological function that resolves spontaneously. However, in some cases, symptoms and signs may evolve over a number of minutes to hours.

3. Concussion may result in neuropathological changes, but the acute clinical symptoms largely reflect a functional disturbance rather than a structural injury and, as such, no abnormality is seen on standard structural neuroimaging studies.

4. Concussion results in a range of clinical signs and symptoms that may or may not involve loss of consciousness. Resolution of the clinical and cognitive symptoms typically follows a sequential course. However, in some cases symptoms may be prolonged.

The clinical signs and symptoms cannot be explained by drug, alcohol, or medication use, other injuries or other comorbidities.

not associated with concussion. However, when a KO happens due to a head blow(s), a concussion is likely on account of some degree of neurological impairment impeding the combatant to get up before the count of 10 is up. Although LOC can occur, other impairments such as disorientation and balance difficulty could prevent a combatant to get up before the 10 count and are consistent with a concussion. However, it is also important to recognise that these impairments could be due to injuries other than concussion (eg, moderate/severe traumatic brain injury and inner ear injury). In mixed martial arts (MMA), $\mathrm{KO}$ is defined as stoppage of the bout by the referee because a combatant has LOC due to a head blow. In this case, it is clear that a concussion has been sustained.

\section{Technical KO (TKO)}

The definition of TKO in combat sports is much more complex as it encompasses a wide variety of reasons why the fight was stopped. In boxing/kickboxing and MMA, a TKO can be given if a doctor stops the fight due concern about an injury (eg, laceration, concussion and orthopaedic injury). In all combat sports, a TKO is also awarded if the combatant or the combatant's corner requests that the bout not continue ('throwing in the towel'). In both boxing/kickboxing and MMA, if the referee determines that a combatant is no longer adequately defending himself or herself, not fighting back or taking a significant amount of 'punishment' (head or body shots), he or she can subjectively call an end to the bout via a TKO. In some formats of boxing/ kickboxing, a TKO can be awarded if the 3 knockdown rule is in place. This rule means that the fight will be stopped if a combatant is knocked down three times within the same round. In all of these instances, there are no mention of signs or symptoms of concussion. Concussion can certainly accompany a TKO, but a TKO does not always have to be associated with a concussion. Lastly, concussion can still occur even if there is no $\mathrm{KO}$ or TKO.
Box 2 Signs and symptoms of concussion ${ }^{5}$

- Somatic symptoms (eg, headache, nausea/vomiting, light/ noise sensitivity and vision problems).

- Cognitive symptoms (eg, foggy feeling, memory problems and difficulty concentrating).

- Emotional symptoms (eg, labile mood).

- Physical signs (eg, loss of consciousness, confusion, difficulty making eye contact, slurred/slow speech and sluggish/change in fighting style).

- Balance impairment (eg, dizziness, difficulty walking and stumbling).

- Behavioural changes (eg, irritability).

- Cognitive impairment (eg, slow to react, slow to answer questions and not oriented to person, location or date/round).

- Sleep/wake disturbance (eg, somnolence and drowsiness).

\section{SIGNS AND SYMPTOMS OF CONCUSSION}

Box 2 lists the signs and symptoms of concussion that could be seen in combat sports. If a combat sports athlete displays one or more of these signs/symptoms, concern for concussion should be raised.

\section{CURRENT PUBLICATIONS REGARDING CONCUSSION IN COMBAT SPORTS}

The current publications on combat sports provide limited data specific to concussion. Numerous publications from the ongoing Professional Fighters Brain Health Study and previous studies have demonstrated an association of increased changes of the brain on imaging and increased cognitive impairment with higher levels of fight exposure (frequency of competing and duration of career) in combat sports athletes. ${ }^{8-15}$ Increase exposure to sparring has also been associated with increased cognitive and balance dysfunction. ${ }^{16}$ However, in these publications, the role of concussion was not clearly defined.

Other publications in combat sports focus on outcomes and injuries sustained in competition. Many of these studies are retrospective assessments of fight records and/or videos. ${ }^{17-23}$ While these studies provide useful information, we recognise the pitfalls of extrapolating the data to concussion. A recent publication highlighted the large discrepancy in fight databases based on differences in documentation practices between sanctioning bodies, experience of the covering ringside physician and proportion of incomplete records. ${ }^{24}$ With regards to video analysis studies, it can only be speculated about whether a concussion had been sustained with no clear signs or detailed postfight physical exam. For these reasons, there are very few high-quality studies available about incidence of concussion in combat sports. A study that examined injuries sustained in professional kickboxing over a 16-year period showed an injury rate of 19.2 concussions per 1000 fight participations. ${ }^{25}$ Another well-designed study that followed elite amateur boxers over a 5 -year period showed an incidence of 0.53 concussions for every 1000 hours of competing/training. ${ }^{26}$

\section{CURRENT PRACTICES IN NON-COMBAT SPORTS}

In non-combat sports, it is universally agreed that, if an athlete is suspected to have suffered a concussion, he or she must be immediately removed from play and evaluated. If a concussion is diagnosed, he or she must not be allowed to return to sport (RTS) on the day of injury. ${ }^{4-7}$ The most recent consensus statement also recommends that athletes with a suspected concussion 
have a thorough evaluation, performed in a distraction free environment (eg, locker room and training room) rather than on the sideline. ${ }^{5}$ Most professional non-combat sports organisations have developed specific protocols for evaluating head injuries during a game. ${ }^{27}$ The overwhelming majority of non-combat sports organisations also require athletes who have sustained a concussion to go through an RTS protocol that consists of a gradual, stepwise increase in physical demands, sports-specific activities and the risk for contact. Many non-combat sports organisations also require medical clearance by a licenced healthcare provider trained in the evaluation and management of concussion prior to full RTS without any restriction. In some cases of professional athletics, an 'unaffiliated physician' who practices independently of the team decides final clearance to RTS. ${ }^{27}$

All consensus statements agree that when an athlete sustains a concussion, an initial cognitive and physical rest period is needed prior to beginning an RTS protocol. However, what is not currently agreed on is the amount or duration of rest needed. Some statements recommend not beginning an RTS progression until the athlete no longer reports any concussion-related symptoms. ${ }^{67}$ Other publications recognise that (1) the current published evidence evaluating the effect of rest following a sports-related concussion is sparse and (2) light exercise may promote recovery after an initial period of rest. ${ }^{45}$ A 2016 publication that summarised a meeting of 37 concussion experts stated that, 'strict rest is contraindicated (after concussion) and may exacerbate the effects of (the) injury'. ${ }^{28}$ This same publication also summarised that concussion symptoms and impairments are treatable and that concussions in which symptoms last a prolonged period of time be managed by a multidisciplinary team with active rehabilitation depending on the individual's clinical profile. ${ }^{28}$ The most recent consensus statement recommends that there should be 'a brief period of rest during the acute phase (24-48 hours) after injury, patients can then be encouraged to become gradually and progressively more active while staying below their cognitive and physical symptom-exacerbation thresholds (eg, activity level should not bring on or worsen their symptoms)', 5

Neuropsychological (NP) testing is one tool that informs sports-related concussion evaluation and management. ${ }^{4-7}$ Various computerised NP testing programmes have been developed. Computerised NP testing, while not as comprehensive as formal 'pencil and paper' NP testing, provides a 'snapshot' of a person's cognitive status. Many non-combat sports organisations use computerised NP testing in RTS protocols. These protocols require athletes to return to his or her preinjury baseline computerised NP testing scores to return to competition. NP testing, however, should not be used as the sole determining factor in management decisions; it may serve as one part of the clinical decision-making process.

\section{CURRENT RTS PRACTICES IN COMBAT SPORTS}

Some combat sports organisations have developed specific written policies regarding intrabout evaluation/management of concussion and RTS after TKO/KO. Specifically, USA Boxing guidelines state that a match should be stopped if a boxer is showing signs and symptoms of concussion. ${ }^{29}$ USA Boxing guidelines also include minimum periods of suspension for $\mathrm{TKO} / \mathrm{KO} .{ }^{30}$

Suspensions in USA Boxing are based on whether there has been an LOC attendant to a $\mathrm{TKO} / \mathrm{KO}$, and the duration of an LOC following a $\mathrm{TKO} / \mathrm{KO}$ as well as number of times a fighter has suffered a TKO/KO with or without LOC (table 1).

Regarding professional combat sports, intrabout evaluation of concussion and RTS after concussion vary from

\begin{tabular}{|c|c|}
\hline \multicolumn{2}{|c|}{ USA Boxing: minimal suspension period after TKO/KO } \\
\hline \multicolumn{2}{|l|}{ Single occurrence of TKO/KO } \\
\hline TKO or KO without LOC & 30-day suspension \\
\hline KO with LOC less than $1 \mathrm{~min}$ & 90-day suspension \\
\hline KO with LOC greater than $1 \mathrm{~min}$ & 180-day suspension \\
\hline \multicolumn{2}{|c|}{ Second occurrence of TKO/KO in a 90-day period after single occurrence suspension } \\
\hline TKO or KO without LOC & 90-day suspension \\
\hline Second KO with LOC less than 1 min & 180-day suspension \\
\hline Second KO with LOC greater than $1 \mathrm{~min}$ & 360-day suspension \\
\hline \multicolumn{2}{|l|}{ Third occurrence of TKO/KO in a 365-day period } \\
\hline TKO or KO without LOC & 12-month suspension \\
\hline Third KO with LOC regardless of time & 18-month suspension \\
\hline
\end{tabular}

KO, knock out; LOC, loss of consciousness; TKO, technical knockout.

state-to-state/commission-to-commission. Some states/commissions have specific written guidelines regarding concussion, but other states/commissions do not, and procedures are followed based on previous precedent and at the discretion of the supervising ringside physician. Furthermore, while in some states/ commissions, ringside physicians are authorised to stop a fight, while in other states/commissions, only the referee is the sole arbitrator of the fight but instructed to stop the fight on medical grounds at the ringside physician's request. Minimum suspensions typically given in professional combat sports are 30 days for a TKO, 60 days for KO without LOC and 90 days for KO with LOC. However, as noted above, these timeframes vary depending on the state/commission. Some states/commissions require a lesser period of suspension if a fight went for a certain number of rounds, win or lose, for example, six or more rounds.

Ultimately, states/commissions often leave medical suspensions to the discretion of the covering ringside physician. Ringside physicians vary greatly in their level of ringside experience and subspecialty medical background leading to variations in postbout recommendations and suspensions. All periods of suspension imposed by states/commissions are enforceable as to competition only and have no effect on what a combatant may do in training/practice/sparring.

Currently, there are no general requirements for combat sports athletes to be evaluated by a licenced healthcare provider specifically trained in the evaluation and management of concussion as a part of medical clearance to RTS following a concussion or $\mathrm{TKO} / \mathrm{KO}$. Although a covering ringside physician may require a neurology clearance evaluation in addition to the period of suspension, such a requirement may not be stringently imposed. Unfortunately, current practices in most jurisdictions rarely require medical clearance to RTS following concussion or TKO/ $\mathrm{KO}$ and generally use the $30 / 60 / 90 /$ day suspensions as outlined above. This risks a combat sports athlete RTS in the absence of a robust medical evaluation.

Currently, no known combat sports organisation requires baseline formal NP or computerised NP testing.

\section{ASSOCIATION OF RINGSIDE PHYSICIANS CONCUSSION MANAGEMENT AND RETURN TO PLAY GUIDELINES FOR COMBAT SPORTS ATHLETES}

Taking into consideration, the above-referenced differences between non-combat sports and combat sports, as well as the absence of uniformity among the jurisdictions that currently 
regulate combat sports, the ARP proposes the following guidelines for concussion management and RTS of a combat sports athlete:

\section{Suspensions and concussion management}

- If a fighter is exhibiting signs of a concussion during a bout, the fight should be stopped. These signs include but are not limited to headache, confusion, blurred/double vision, nausea/vomiting and balance/gait issues (box 2).

- If a combat sports athlete sustains a TKO secondary to blows to the head, it is recommended that he or she be suspended from competition for a minimum of 30 days. It is also recommended that the fighter refrain from sparring for 30 days as well.

- If a combat sports athlete sustains a KO without LOC secondary to blows to the head, it is recommended that he or she be suspended from competition for a minimum of 60 days. It is also recommended that the fighter refrain from sparring for 60 days as well.

- If a combat sports athlete sustains a KO with LOC secondary to blows to the head, it is recommended that he or she be suspended from competition for a minimum of 90 days. It is also recommended that the fighter refrain from sparring for 90 days as well.

- All combat sports athletes, including the winners, should be evaluated for signs and symptoms of concussion postbout. Evaluation should be performed immediately postbout ringside but also later repeated in a quieter, controlled environment (eg, dressing room).

- Combat sports athletes may participate in non-contact training and conditioning 1 week after sustaining a concussion or loss via $\mathrm{TKO} / \mathrm{KO}$ secondary to head strikes, provided his or her symptoms are improving and do not increase in severity with activity. A gradual activity progression of increased intensity is recommended, starting with light aerobic activity progressing to more rigorous/combat sports-specific activity and finally sparring when symptoms have completely resolved (box 3 ).

- Under no circumstances should a combat sports athlete compete or engage in sparring activity or competition if he or she is experiencing signs and symptoms of concussion.

\section{Specialist evaluation}

- In addition to the above-mentioned periods of suspension, we recommended that a combat sports athlete's suspension continue until a specialist physician trained in concussion management clears the fighter. Specialist physicians trained in concussion management include neurologists, neurosurgeons and primary care sports medicine physicians.

\section{Box 3 'Red flag' signs and symptoms of serious brain}

\section{injury}

Glasgow Coma Scale $<15$

- Suspected open, depressed or basal skull fracture.

- Cerebrospinal fluid coming out of nose or ears.

- Post-traumatic seizure.

- Focal neurological deficit.

>1 episode of vomiting since the head injury.

- Pupillary abnormality.

- Progressive increase of concussion somatic symptoms.

- Deterioration of mental status/overall condition.

\section{Other baseline testing}

- We recommended that all combat sports athletes undertake a validated NP evaluation with the initial test serving as a baseline. If possible, vestibular/ocular and balance baseline testing is also recommended. Repeat baseline testing should occur annually. If there is any decline, it is recommended that a physician trained in brain injuries and concussion management evaluate the athlete.

\section{Education}

- We recommended that all combat sports athletes and their coaches/trainers be educated and trained to recognise the signs and symptoms of concussion. If a combat sports athlete is experiencing any signs or symptoms of a concussion during training or competition, he or she should remove themselves from contact activities and seek immediate evaluation by a healthcare professional.

\section{DISCUSSION OF GUIDELINES}

The ARP recognises the rapid advancements in concussion management over the recent years and its adaptation into non-combat sports. This has created a safer environment and may lead to a decrease in morbidity associated with sports-related head trauma. Current non-combat sports RTS protocols do not allow an athlete to return to competition/contact if he or she is exhibiting any concussion symptoms. If a non-combat sports athlete suffers an injury and is showing signs or symptoms of concussion, he or she is removed from competition immediately, with no same-day RTS. The ARP agrees that this practice should also be employed in combat sports. If a combat sports athlete is exhibiting signs or symptoms of concussion during a bout (box 2), he or she should be evaluated by the covering ringside physician, and if a concussion is suspected, the bout should be immediately stopped on medical grounds (TKO).

Often, emphasis in suspensions and medical treatment is focused on the loser of the bout, or the one who suffers a $\mathrm{KO}$ or TKO. The ARP recommends that all combat sports athletes, including the winner, be evaluated for signs and symptoms of concussion and should be managed and treated accordingly. Evaluation should be performed immediately postbout ringside. A repeat evaluation should be performed postbout in a quieter, controlled setting such as the dressing room. Repeat evaluation is necessary to ensure that combatants do not display delayed onset signs and symptoms of concussion. It is not unreasonable for the covering ringside physician to consider a minimum suspension of 30 days for all fighters, win/lose/draw, if the fighter has been through a particularly long, gruelling, difficult bout.

The covering ringside physician (and other covering healthcare providers) should also be aware and evaluate fighters, postbout, for 'red flag' signs (box 3). Presence of these 'red flag' signs and symptoms should raise concern that a more serious brain injury has been sustained and the fighter should be emergently transported to a high-level emergency facility for screening and management of a potentially life-threatening condition.

Non-combat sports allow athletes to return to competition/ contact when concussion symptoms resolve, and the athlete has tolerated a stepwise progression of activity without return of symptoms. The ARP is also in agreement with this premise. However, due to the nature and objective of combat sports, it is recommended that combat sports athletes should also wait a minimum specified amount of time (30/60/90 days) until return to contact. Return to fight protocols should be specific to the athlete's combat sport. 


\begin{tabular}{lcl}
\hline Table 2 & Return to fighting protocol \\
\hline Phase 1: return to general fitness \\
\hline Step 1 & Light aerobic activity & $\begin{array}{l}\text { Stationary biking and elliptical, incline walking: } \\
\text { gradually escalating heart rate and monitoring } \\
\text { through perceived exertion and/or HRM. }\end{array}$ \\
Step 2 & $\begin{array}{l}\text { Moderate aerobic } \\
\text { activity }\end{array}$ & $\begin{array}{l}\text { Jogging, swimming: escalating heart rate to } \\
\text { moderate and high-demand activity through } \\
\text { HRM or perceived exertion; assess high-level } \\
\text { vestibular functioning. }\end{array}$ \\
Step 3 & Sport-specific activity & $\begin{array}{l}\text { Sprinting, mitts, bag/footwork, walk-through } \\
\text { grappling and so on; increasing duration; no } \\
\text { partner work. Begin resistance training. }\end{array}$ \\
\hline Phase 2: return to non-contact fighting activities \\
\hline Step 1 & $\begin{array}{l}\text { Bag/mitt work with } \\
\text { movement }\end{array}$ & $\begin{array}{l}\text { Tests fighter ability to punch and/or kick in } \\
\text { multiple planes while testing vestibular and } \\
\text { visual systems. }\end{array}$ \\
Step 2 & Shadow boxing/drills & $\begin{array}{l}\text { Reintroduces fighter to sport environment and } \\
\text { re-establishes footwork in ring parameter and } \\
\text { surface. }\end{array}$ \\
\hline Step 3 & $\begin{array}{l}\text { One-sided sparring } \\
\text { and grappling }\end{array}$ & $\begin{array}{l}\text { Fighter begins to spar without the concern of } \\
\text { contact. Reacts to opponent's movements and } \\
\text { begins to get timing back for punches, kicks } \\
\text { and body position. }\end{array}$ \\
\hline
\end{tabular}

Phase 3: return to contact/sparring fighting activities

May only advance to this phase when concussion symptoms have completely resolved

\begin{tabular}{lll}
\hline Step 1 & $\begin{array}{l}\text { Sparring: short } \\
\text { duration }\end{array}$ & $\begin{array}{l}\text { First step of live sparring. Rounds of short } \\
\text { duration with long breaks. Number of rounds } \\
\text { is small to begin with and then can increase } \\
\text { as tolerated. }\end{array}$ \\
Step 2 & $\begin{array}{l}\text { Sparring: longer } \\
\text { duration }\end{array}$ & $\begin{array}{l}\text { Rounds at this step begin to lengthen in } \\
\text { duration while breaks between rounds } \\
\text { shortens. Number of rounds can also increase } \\
\text { as fatigue allows. }\end{array}$ \\
Step 3 & $\begin{array}{l}\text { Sparring: normal } \\
\text { parameters }\end{array}$ & $\begin{array}{l}\text { Full return to normal training. Return to normal } \\
\text { rounds and time limits based on sport and } \\
\text { next potential bout. Fighter should be able to } \\
\text { tolerate normal parameters of training/sparing } \\
\text { and is training as normal without a return of } \\
\text { symptoms. }\end{array}$ \\
\hline
\end{tabular}

An initial period of 1 week should occur prior to beginning phase 1 .

${ }^{*}$ Adapted from Nalepa et al. ${ }^{32}$

Nalepa $e t a l^{32}$ have proposed recommendations with regard to return to fight protocols (table 2). This protocol is broken down into three phases: phase 1: return to general fitness; phase 2: return to non-contact fighting activities; and phase 3: return to contact fighting activities/sparring. It is recommended that a combat sports athlete should not begin phase 1 for at least 1 week after sustaining a concussion and/or loss via $\mathrm{TKO} / \mathrm{KO}$ secondary to head strikes. This initial rest phase is the cornerstone of concussion management and allows for acute symptoms to resolve. ${ }^{4}$ Combat sports athletes may progress from phase 1 to phase 2 provided symptoms do not increase in severity. The combat sports athlete should perform one step per day, waiting a minimum of an additional 24 hours before moving on to the next step. If symptoms do not increase over that 24-hour period, he or she may move on to the next step. The athlete should not advance if concussion symptoms increase in that 24-hour period. If symptoms increase, he or she should go back to the prior step where symptoms did not increase. Under no circumstance should a combat sports athlete progress to phase 3 if he or she continues to exhibit concussion symptoms. Athletes who remain symptomatic for greater than 10 days should be seen by a physician trained in concussion management to monitor symptoms, initiate treatments and recommend activity progression.

For phases 1 and 2, there should be at least 24 hours (or longer) for each step of the progression. If any symptoms worsen during exercise, the athlete should go back to the previous step. Phase 3 should be started when concussion symptoms have resolved, and the athlete has completed phases 1 and 2 . If symptoms are persistent (eg, more than 10 days), the athlete should be referred to a healthcare professional that is an expert in the management of concussion.

The majority of non-combat sports concussion protocols throughout the amateur and professional levels require evaluation and clearance by a healthcare provider trained in the management of concussion before return to contact/competition. The ARP also believes such a practice should be applied in combat sports. If a combat sports athlete is experiencing concussion signs and symptoms $30 \mathrm{~min}$ after his or her bout, or lost his or her bout by $\mathrm{KO}$ via strikes to the head, it is most likely the athlete has sustained a concussion. Accordingly, he or she should be evaluated and cleared by a healthcare provider skilled in concussion management before return to contact/competition. The specialist clearance should be in addition to the above-referenced recommended minimal day suspensions.

NP baseline testing is a helpful tool in making decisions regarding a return to fighting in combat sports athletes who have sustained a concussion. NP testing is also helpful for screening of neurological decline over the course of a combatant's career. Computerised NP baseline testing is now widely available and affordable. For these reasons, the ARP recommends that all combat sports athletes undergo a validated, supervised and reproducible NP baseline test. Baseline vestibular/ocular and balance testing is a recommended adjunct for the same reasons.

Finally, none of these guidelines can be enforced outside of competition. Only the combat sports athlete and his or her coaches/trainers know what happens inside the gym and during training. Hence, athletes and their coaches/trainers should possess a thorough knowledge base regarding concussion and its signs/symptoms (box 2). Unfortunately, when there has been a tragic outcome in the ring, it is often revealed that the combat sports athlete suffered a head injury in training that was not divulged to the physician. If a combat sports athlete sustains an injury and is experiencing any signs or symptoms of a concussion during training or competition, he or she should be encouraged to remove themselves from contact activities and seek evaluation by a healthcare professional.

\section{QUALIFYING STATEMENT}

These guidelines are recommendations designed to assist ringside physicians, fighters, trainers, promoters, sanctioning bodies, governmental bodies and others in making decisions. These recommendations may be adopted, modified or rejected according to clinical needs and constraints and are not intended to replace local commission laws, regulations or policies. We hope that these guidelines will serve to augment locally agreed policies already in place. In addition, it should be understood that the guidelines developed by the ARP are not intended as standards or absolute requirements, and their use cannot guarantee any specific outcome. Guidelines are subject to revision as warranted by the evolution of medical knowledge, technology and practice. They provide basic recommendations that are supported by synthesis and analysis of the current literature, expert and practitioner opinion, commentary and clinical feasibility. 


\section{Author affiliations}

'Department of Sports Medicine, Orthopaedic Specialists of North Carolina, Raleigh, North Carolina, USA

${ }^{2}$ Campbell University School of Osteopathic Medicine, Buies Creek, North Carolina USA

${ }^{3}$ Department of Neurology, New York-Presbyterian Hospital, New York City, New York, USA

${ }^{4}$ Memorial Hermann Healthcare System, Houston, Texas, USA

${ }^{5}$ Baylor College of Medicine Department of Family and Community Medicine, Houston, Texas, USA

${ }^{6}$ Aneurin Bevan University Health Board, Newport, UK

${ }^{7}$ Royal College of Surgeons in Ireland Faculty of Sports and Exercise Medicine, Dublin, Ireland

${ }^{8}$ Essentia Health, Duluth, Minnesota, USA

${ }^{9}$ University of Minnesota Medical School - Duluth Campus, Duluth, Minnesota, USA

${ }^{10}$ Association of Ringside Physicians - Legal Counsel, Baltimore, Maryland, USA

${ }^{11}$ INTEGRIS Southwest Medical Center, Oklahoma City, Oklahoma, USA

${ }^{12}$ Desert Regional Medical Center, Palm Springs, California, USA

${ }^{13}$ White Plains Hospital Center, White Plains, New York, USA

${ }^{14}$ Metrolina Orthopedic and Sports Medicine Clinic, Charlotte, North Carolina, USA

${ }^{15}$ Phoenix VA Health Care System, Phoenix, Arizona, USA

${ }^{16} \mathrm{Centre}$ for the Study of Traumatic Encephalopathy, Boston University School of Medicine, Boston, Massachusetts, USA

${ }^{17}$ Department of Neurosurgery, Boston University School of Medicine, Boston, Massachusetts, USA

${ }^{18}$ The Burke Rehabilitation Hospital, New York City, New York, USA

${ }^{19}$ Voluntary Anti-Doping Association, Las Vegas, Nevada, USA

${ }^{20}$ Mood and Anxiety, Psychiatry, University of Maryland Baltimore Medical School, Baltimore, Maryland, USA

${ }^{21}$ George Washington University School of Medicine and Health Sciences, Washington, District of Columbia, USA

${ }^{22}$ Connecticut Children's Medical Center, Hartford, Connecticut, USA

${ }^{23}$ Elite Sports Medicine, Farmington, Connecticut, USA

${ }^{24}$ Jupiter, Florida, USA

${ }^{25}$ Cape Regional Health System, Cape May Court House, New Jersey, USA

${ }^{26}$ New York, USA

${ }^{27}$ Yeshiva University Albert Einstein College of Medicine, Bronx, New York, USA

${ }^{28}$ Saint Agnes Medical Center, Fresno, California, USA

${ }^{29}$ Loyola University Chicago Stritch School of Medicine, Maywood, Illinois, USA

${ }^{30}$ Cedars-Sinai Medical Center, Los Angeles, California, USA

${ }^{31}$ Fort Lauderdale, USA

${ }^{32}$ St. Augustine, Trinidad and Tobago

Contributors Substantial contributions to the conception or design of the work, or the acquisition, analysis or interpretation of data: JN, NKS, RT, RM and DM. Drafting the work or revising it critically for important intellectual content: JN, NKS, RT, RM, DM, LL, EA, RW, JE, PR, RCC, BJ, MG, JWS and JG. Grammar editing: BS. Final approval of the version published: all authors. Agreement to be accountable for all aspects of the work in ensuring that questions related to the accuracy or integrity of any part of the work are appropriately investigated and resolved: all authors.

Funding The authors have not declared a specific grant for this research from any funding agency in the public, commercial or not-for-profit sectors.

Competing interests PR serves as the CMO for the Retired NFLPA and as CTO of Premier Biomedical. RCC serves as a consultant to the NFL, Concussion Legacy Foundation and to NOCSAE. A version of this statement is posted on the ARP website: http://www.ringsidearp.org/

Patient consent Not required.

Provenance and peer review Not commissioned; externally peer reviewed.

Open access This is an open access article distributed in accordance with the Creative Commons Attribution Non Commercial (CC BY-NC 4.0) license, which permits others to distribute, remix, adapt, build upon this work non-commercially, and license their derivative works on different terms, provided the original work is properly cited, appropriate credit is given, any changes made indicated, and the use is non-commercial. See: http://creativecommons.org/licenses/by-nc/4.0/.

\section{REFERENCES}

1 Aubry M, Cantu R, Dvorak J, et al. Summary and agreement statement of the 1st International Symposium on Concussion in Sport, Vienna 2001. Clin J Sport Med 2002:12:6-11.
2 McCrory P, et al. Summary and agreement statement of the 2nd International conference on concussion in Sport, Prague 2004. Br J Sports Med 2005:39:i78-86.

3 McCrory P, Meeuwisse W, Johnston K, et al. Summary and agreement statement of the 3rd International Symposium on Concussion in Sport, held in Zurich, November 2008. Br J Sport Med;2009:176-84.

4 McCrory P, Meeuwisse WH, Aubry M, et al. Consensus statement on concussion in sport: the 4th international conference on concussion in sport held in Zurich, November 2012. Br J Sports Med;2013; 47:250-8.

5 McCrory P, Meeuwisse WH, Dvorak J, et al. Consensus statement on concussion in sport - the 5th international conference on concussion in sport held in Berlin, October 2016. Br J Sport Med;2017; 51:838-47.

6 Harmon KG, Drezner J, Gammons M, et al. American Medical Society for Sports Medicine Position Statement. Clinical Journal of Sport Medicine 2013;23:1-18.

7 Broglio SP, Cantu RC, Gioia GA, et al. National Athletic Trainers' Association position statement: management of sport concussion. J Ath/ Train 2014;49:245-65.

8 Bernick C, Banks S, Phillips M, et al. Professional fighters brain health study: rationale and methods. Am J Epidemiol 2013;178:280-6.

9 Bernick C, Banks S. What boxing tells us about repetitive head trauma and the brain. Alzheimers Res Ther 2013:5:23.

10 Bernick C, Banks SJ, Shin W, et al. Repeated head trauma is associated with smaller thalamic volumes and slower processing speed: the Professional Fighters' Brain Health Study. Br J Sports Med 2015:49:1007-11.

11 Chappell MH, Ulu?? AM, Zhang L, et al. Distribution of microstructural damage in the brains of professional boxers: A diffusion MRI study. Journal of Magnetic Resonance Imaging 2006;24:537-42

12 Mishra VR, Zhuang X, Sreenivasan KR, et al. Multimodal mr imaging signatures of cognitive impairment in active professional fighters. Radiology 2017;285:555-67.

13 Ravdin LD, Barr WB, Jordan B, et al. Assessment of cognitive recovery following sports related head trauma in boxers. Clinical Journal of Sport Medicine 2003;13:21-7.

14 Shin W, Mahmoud SY, Sakaie K, et al. Diffusion measures indicate fight exposurerelated damage to cerebral white matter in boxers and mixed martial arts fighters. American Journal of Neuroradiology 2014:35:285-90.

15 Zhang L, Ravdin L, Relkin N, et al. Increased diffusion in the brain of professional boxers: a preclinical sign of traumatic brain injury? AJNR Am J Neuroradiol 2003;1:52-7.

16 Stiller JW, Yu SS, Brenner LA, et al. Sparring and neurological function in professional boxers. Front Public Health 2014:2:1-6.

17 Buse GJ. No holds barred sport fighting: a 10 year review of mixed martial arts competition. Br J Sports Med 2006:40:169-72

18 Hutchison MG, Lawrence DW, Cusimano MD, et al. Head trauma in mixed martial arts. Am J Sports Med 2014;42:1352-8.

19 Jensen AR, Maciel RC, Petrigliano FA, et al. Injuries sustained by the mixed martial arts athlete. Sports Health 2017;9:64-9.

20 Lystad RP, Gregory K, Wilson J. The epidemiology of injuries in mixed martial arts. Orthop J Sports Med 2014;2:1-10.

21 McClain R, Wassermen J, Mayfield C, et al. Injury profile of mixed martial arts competitors. Clinical Journal of Sport Medicine 2014;24:497-501.

22 Ngai KM, Levy F, Hsu EB. Injury trends in sanctioned mixed martial arts competition: a 5-year review from 2002 to 2007. Br J Sports Med 2008;42:686-9.

23 Otten MH, Ghazarian N, Boura J. Ultimate fighting championship injuries: a two-year retrospective fight injury study. Osteopath Family Physician 2015;7:13-18.

24 Thomas R, Thomas B. Systmatic review of injuries in mixed martial arts. Phys Sportsmed 2018;25:1-13.

25 Zazryn TR, Finch C, McCrory P. A 16 year study of injuries to professional kickboxers in the state of Victoria, Australia. Br J Sports Med 2003:37:448-51.

26 Loosemore $M$, Lightfoot J, Palmer-Green $D$, et al. Boxing injury epidemiology in the Great Britain team: a 5-year surveillance study of medically diagnosed injury incidence and outcome. Br J Sports Med 2015;49:1100-7.

27 NFL Health Playbook. National Football League head, neck and spine committee's protocols regarding return to participation following concussion. http://static.nfl.com/ static/content/public/photo/2014/08/21/0ap3000000381608.pdf (accessed 01 Sep 2016).

28 Collins MW, Kontos AP, Okonkwo DO, et al. Statements of agreement from the Targeted Evaluation and Active Management (TEAM) Approaches to treating concussion meeting held in Pittsburgh, October 15-16, 2015. Neurosurgery 2016:79:912-29.

29 USA Boxing. Medical Handbook and Medical Rules of AIBA Open Boxing, 2016

30 USA Boxing. USA Boxing national rule book: effective, 2017.

31 Davis T, Ings A. Head injury: triage, assessment, investigation and early management of head injury in children, young people and adults (NICE guideline CG 176). Archives of disease in childhood - Education \& practice edition 2015;100:97-100.

32 Nalepa B, Alexander A, Schodrof S, et al. Fighting to keep a sport safe: toward a structured and sport-specific return to play protocol. Phys Sportsmed 2017:45:1-6. 